\title{
Impaired Sensorimotor Gating in Unmedicated Adults with Obsessive-Compulsive Disorder
}

\author{
Susanne E Ahmari*, ${ }^{1,2,3}$, Victoria B Risbrough ${ }^{4}$, Mark A Geyer ${ }^{4}$ and H Blair Simpson ${ }^{1,3}$ \\ 'Department of Psychiatry, Columbia University, College of Physicians \& Surgeons, New York, NY, USA; 2Division of Integrative Neuroscience, \\ New York State Psychiatric Institute, New York, NY, USA; ${ }^{3}$ Division of Clinical Therapeutics, New York State Psychiatric Institute, New York, NY, \\ USA; ${ }^{4}$ Department of Psychiatry, University of California, San Diego, CA, USA
}

\begin{abstract}
Functional and structural imaging studies suggest that obsessive-compulsive disorder (OCD) symptoms arise from dysfunction in corticostriato-thalamo-cortical circuits. It has therefore been hypothesized that neurophysiological tasks subserved by these circuits should be abnormal in OCD patients. One neurocognitive probe associated with this circuitry is prepulse inhibition (PPI) of the acoustic startle response. PPI deficits are thought to reflect abnormalities in processing and integration of sensory and motor information. Two prior studies found that OCD patients had PPI deficits at single prepulse (PP) intensities. However, most patients in these studies were taking psychotropic medications at the time of PPI testing, and preclinical studies have demonstrated effects of psychotropic medications on PPI. We examined PPI in 22 unmedicated $\mathrm{OCD}$ patients and 22 matched healthy controls at three different PP intensities (74, 78, and $86 \mathrm{~dB})$. OCD patients had significantly less PPI across all three PP intensities compared with controls. Exploratory analyses indicated that OCD patients with a history of tics had lower levels of PPI. Our results demonstrate that unmedicated OCD patients have impaired sensorimotor gating as measured by PPI. This indicates that PPI deficits are present in OCD patients and are not the result of medication effects. Our findings also suggest that OCD patients with a history of tics may have greater impairment in sensorimotor gating than the general OCD population. Future studies should be designed to examine whether PPI deficits characterize tic-related OCD. Neuropsychopharmacology (2012) 37, 1216-1223; doi:10.1038/npp.201 I.308; published online 4 January 2012
\end{abstract}

Keywords: obsessive-compulsive disorder; prepulse inhibition; sensorimotor gating; serotonin reuptake inhibitors; acoustic startle; tic disorder

\section{INTRODUCTION}

Obsessive-Compulsive Disorder (OCD) is a severe, chronic psychiatric disorder with 2-3\% prevalence worldwide (Robins et al, 1984; Kessler et al, 2005). Despite the public health burden of OCD (Murray and Lopez, 1996), relatively little is known about its neurobiology. Functional and structural imaging studies suggest that OCD symptoms arise from dysfunction in brain circuits that include the orbitofrontal cortex, anterior cingulate cortex, striatum, and thalamus (Maia et al, 2008; Hammer et al, 2009). It is thus predicted that performance on neurophysiological tasks subserved by these circuits will be abnormal in people with OCD. Sensorimotor gating as measured by prepulse inhibition (PPI) is one well-established method for probing functional status of cortico-striato-pallidal circuitry in humans and rodents (Geyer and Dulawa, 2003). Our study is therefore aimed at determining whether PPI is abnormal in unmedicated OCD patients compared with healthy controls.

*Correspondence: Dr SE Ahmari, Department of Psychiatry, Columbia University, College of Physicians \& Surgeons, New York, NY I00322695, USA, Tel: + I 212543 5036, Fax: + I 2125435074

E-mail: sea2103@columbia.edu

Received 3 August 2011; revised 30 October 2011; accepted 31 October 2011
Sensorimotor gating refers to the process by which a neural system screens or 'gates' extraneous external (sensory) and internal (cognitive, motor) information from higher order processing and subsequent responses, presumably to enable uninterrupted processing of the most salient aspects of the external and internal environment (Butler et al, 1990). Thus, sensorimotor gating mechanisms are thought to prevent information overload, and facilitate mental and behavioral integration. Reductions in sensorimotor gating are theorized to reflect deficient central inhibitory functioning and underlie symptoms of several neuropsychiatric disorders, including the obsessions and compulsions of OCD (Swerdlow et al, 1993; Hoenig et al, 2005). Specifically, obsessions are thought to arise from the inability to inhibit undesired thoughts and images, and compulsions from the inability to inhibit repetitive acts or reactions to uncontrollable obsessive thoughts.

One operational measure of sensorimotor gating that has been well validated in animal and human studies is PPI (Geyer et al, 2001). PPI is the reduction in startle reflex that occurs when a startling stimulus ('pulse') is preceded by a barely detectable prepulse (PP) stimulus; the sensory PP thus inhibits or 'gates' the motor startle reflex (Graham, 1975). Evidence from rodent studies has indicated that PPI is mediated at the level of the pons in the pedunculopontine 
nucleus (Fendt et al, 2001), and modulated by distributed forebrain circuits involving the prefrontal cortex, basal ganglia, thalamus, hippocampus, amygdala, and subpallidal efferents to the pedunculopontine nucleus (Koch and Schnitzler, 1997; Swerdlow and Geyer, 1998; Swerdlow et al, 2001; Geyer et al, 2001). Thus, brain pathways implicated in OCD overlap with those that modulate PPI, particularly at the level of fronto-striatal and striato-pallidal connectivity.

Three studies have examined whether OCD patients have sensorimotor gating deficits as measured by PPI of the acoustic startle response. In a preliminary study, Swerdlow et al (1993) found that PPI was impaired in 11 OCD patients compared with 13 controls at one PP intensity $(74 \mathrm{~dB})$. Hoenig et al (2005) replicated these findings in a larger sample ( $N=30 \mathrm{OCD}, N=30$ controls), although a different PP intensity was found to be affected ( $86 \mathrm{~dB})$. Finally, de Leeuw et al (2010) did not identify PPI deficits at any of three PP intensities $(74,78$, and $86 \mathrm{~dB}$ ) in $25 \mathrm{OCD}$ patients and 25 controls, although methodological differences between this study and the others (eg, methods for PPI measurement, length of medication-free period, and subject selection/ screening) may have had a role.

The studies conducted by Swerdlow et al (1993) and Hoenig et al (2005) suggest that PPI may be impaired in people with OCD. This fits with prior studies indicating that PPI is subserved by many of the same brain regions that have been hypothesized to be dysfunctional in OCD (Swerdlow et al, 2001; Geyer et al, 2001). However, in both studies, most patients were on medication at the time of testing. As there are known effects of psychotropic medications on PPI (Swerdlow et al, 1994; Quednow et al, 2004; Shanahan et al, 2009), these studies were unable to determine whether the observed PPI deficits were because of medication effects. Moreover, neither study examined whether PPI deficits are associated with key clinical features of OCD hypothesized to have different neurobiological substrates (eg, symptom dimensions, tic-related OCD, and obsessions $v s$ compulsions).

To address this gap in the literature, we examined PPI at three different PP intensities in 22 unmedicated OCD patients and 22 matched healthy controls. We hypothesized that unmedicated OCD patients would have PPI deficits compared with controls. We also explored whether PPI levels in OCD patients were associated with clinical features of OCD. In particular, we examined associations between PPI levels and OCD severity (as greater deficits in sensorimotor gating might lead to more obsessions and compulsions), history of tics (as PPI deficits have been found in patients with Tourette's syndrome (Castellanos et al, 1996; Swerdlow et al, 2001)), age of OCD onset (as pathophysiology of early and late onset OCD may differ (Dickel et al, 2006; Huyser et al, 2009; Simpson et al, 2011), and OCD symptom dimensions (as different OCD dimensions have been associated with different neural substrates (Leckman et al, 2010)).

\section{METHODS}

\section{Subjects}

In all, 25 OCD subjects and 24 healthy controls were screened. A hearing test was performed with an audiometer
(Welch Allyn) to ensure hearing was within normal limits. Testing was performed bilaterally with tones of 500, 1000, 3000 , and $6000 \mathrm{~Hz}$ at $35 \mathrm{~dB}$. Subjects who failed to hear any individual frequency in either ear were not studied further (two OCD subjects; one healthy control). Two additional subjects were excluded from the final analyses (one OCD subject; one healthy control) because they had no startle response (see 'PPI Data Processing' for further details). Thus, the final study population consisted of 22 OCD subjects and 22 matched healthy controls ( 12 males/10 females per group). All participants provided written signed informed consent after explanation of study procedures. The study protocol was approved by the Internal Review Board of New York State Psychiatric Institute/Columbia University.

\section{Screening Procedures and Clinical Assessment}

Eligible subjects were between ages 18 and 55, had no significant medical problems (including hearing loss), were not pregnant or nursing, had no current or past neurological disorder (other than tic disorder), and were free of psychoactive medications. Because of known effects of hormonal state on PPI (Swerdlow et al, 1997; Jovanovic et al, 2004), we excluded women with uncertain hormonal state (ie, using hormonal contraceptives, pregnant, or postmenopausal), and measured PPI in the early follicular phase of the menstrual cycle (day 1-10). OCD subjects met OCD criteria from the Diagnostic and Statistical Manual of Mental Disorders (DSM-IV) for at least 1 year, and had clinically significant symptoms (Yale-Brown ObsessiveCompulsive Scale (Y-BOCS) score $\geqslant 16$ ). They had no other current Axis I psychiatric disorder apart from one subject with chronic tic disorder, one subject with social phobia, and one subject with panic disorder; and no lifetime diagnosis of psychotic disorder, bipolar disorder, eating disorder, mental retardation, or substance abuse/dependence. None were receiving OCD treatment at the time of study participation (either medication or cognitive-behavioral therapy (CBT)), and OCD subjects were also required to be free of all psychotropic medications for at least 4 months. One subject was in therapy (once-weekly psychodynamic psychotherapy). Healthy controls had no current or past DSM-IV Axis I or Axis II disorder, and had never taken psychotropic medication; in addition, none had a family history of OCD, psychotic disorders, Tourette's syndrome, or autism (all of which have been associated with PPI abnormalities) as assessed by Family History Screen (Weissman et al, 2000). Healthy controls were recruited to be matched to OCD subjects on age, gender, ethno-racial groups, verbal IQ (measured using the National Adult Reading Test (NART)), and smoking status (one healthy control and one OCD subject smoked, but did not meet criteria for nicotine dependence) (Nelson and O'Connell, 1978).

Psychiatric diagnoses were made by clinical interview and confirmed with Structured Clinical Interview for DSM-IV, parts I and II (Ekselius et al, 1994; First and Spitzer, 1996). Medical health was confirmed in all subjects by physical exam, electrocardiogram, blood tests, and urinalysis. Urine pregnancy test and urine drug screen (Medical Disposables) were used to confirm that subjects were not pregnant or 
taking illicit substances, opiates, or benzodiazepines on the day of testing. The Yale Global Tic Severity Scale was used to assess tics (Leckman et al, 1989). YBOCS checklist and clinical evaluation were used to assess grooming behaviors. OCD and depressive severity were assessed by a trained rater using the Y-BOCS (scale range: $0-40 ; 16$ and above is considered clinically significant OCD (Goodman et al, 1989a,b)), and the Hamilton Depression Rating Scale (HAM-D, 17-item, scale range: 0-50 (Hamilton, 1960)). The Y-BOCS checklist was used to generate scores for each OCD patient along five different symptom dimensions (contamination and cleaning, taboo thoughts, doubt and checking, symmetry and ordering, and hoarding), using the procedure recommended by Pinto et al (2007, 2008, 2009).

\section{PPI Measurement}

Standard procedures for measuring PPI were used (Braff et al, 2001). Briefly, on the testing day, subjects were asked to refrain from smoking or drinking coffee for at least $1 \mathrm{~h}$ before testing. During testing, the eyeblink component of the acoustic startle response was assessed by recording orbicularis oculi electromyogram (EMG) after loud startling noises were presented using an EMG startle system (EMGSR-LAB, San Diego Instruments, San Diego, CA, USA) for digitization and analysis. To record EMG, two miniature silver/silver chloride electrodes were positioned below and lateral to the right eye over the orbicularis oculi muscle of each subject, and a ground electrode was placed on the right mastoid; electrode resistances were kept $<10 \mathrm{k} \Omega$. After placement of EMG electrodes, subjects were seated comfortably in a chair in a well-lit acoustically isolated room, and asked to look straight ahead and keep their eyes open during testing (ie, blink if necessary, but not fall asleep). A fixation area was provided on the wall, and subjects were told to look at this area to minimize head and neck movements. Headphones were placed on their head. They were seated out of sight of recording equipment and the experimenter, who remained in the room to monitor subjects during the experiment. Before the test began, subjects were informed that they would hear white noise interrupted by loud bursts of noise through the headphones during the testing period. Acoustic startle stimuli were presented binaurally through headphones. Setup and testing lasted $\sim 35 \mathrm{~min}$.

PPI session parameters were similar to those used by Hoenig et al (2005), with two exceptions: we added habituation trials (to determine if OCD subjects had different habituation responses than controls), and we excluded the $72 \mathrm{~dB}$ PP condition (which did not yield robust PPI in Hoenig et al, 2005). Thus, each session consisted of three PP trials, a pulse alone (PA) trial, and a no-stimulus trial $(70 \mathrm{~dB})$ pseudorandomly presented in five blocks of 12 trials, with a single additional PA trial at the beginning of the session, and with $5 \mathrm{PA}$ trials to measure habituation at session beginning and end (71 trials total $=5 \mathrm{PA}$ [Habituation $\left._{1}\right]+1 \mathrm{PA}+60[5 \times 12]$ block trials $+5 \mathrm{PA}\left[\right.$ Habituation $\left.\left._{2}\right]\right)$. Each session began with a 4-min acclimation period of $70 \mathrm{~dB}$ white noise that continued as background noise throughout the session. PA trials were comprised of a $116 \mathrm{~dB} 40 \mathrm{msec}$ white noise burst over $70 \mathrm{~dB}$ background.
PP trials consisted of $20 \mathrm{msec}$ white noise bursts of 74, 78, or $86 \mathrm{~dB}$, with a fixed onset $120 \mathrm{msec}$ before PA presentation. As in Hoenig et al (2005), intertrial intervals were chosen to vary between $10-20 \mathrm{~s}$, with an average of $15 \mathrm{~s}$. The amplifier gain was kept constant for all subjects. EMG was recorded from the onset of the acoustic startle stimulus for $250 \mathrm{msec}$. Sampling rate was $1000 \mathrm{~Hz}$.

\section{PPI Data Processing}

Standard methods for processing PPI data were used (Braff et al, 2001). Voluntary and spontaneous blinks were excluded using registration parameters and exclusion criteria described by Braff et al (2001). Baseline to peak startle magnitude and peak latency were calculated using commercially available software (SRRED, San Diego Instruments). As mentioned above, one OCD subject and one healthy control were classified as 'non-startlers' (mean PA amplitude of the first block was $<3$ times the average amplitude of no-stimulus trials; see Braff et al (2001) for details), and were therefore excluded from further analysis. Viable PPI data were therefore collected from 22 OCD subjects and 22 matched healthy controls.

Latency to response onset was defined as a shift of six digital units from baseline occurring $18-100 \mathrm{msec}$ after presentation of the startle stimulus, and latency to response peak as the point of maximal amplitude that occurred within $150 \mathrm{msec}$ after presentation of the startle stimulus. A computer algorithm was used to determine maximal amplitude. Trials were rejected if onset-to-peak latency was $>95 \mathrm{msec}$ (indicating that the response was not temporally linked to the stimulus such as might occur with voluntary or spontaneous eyeblinks), or if there were baseline shifts $>90$ units (indicating background noise in signal typically due to subject movement).

\section{Outcome Measures}

The main outcome measure was percent PPI, calculated by relating mean startle magnitude of each $\mathrm{PP}$ condition to mean PA magnitude $[(\mathrm{PA}-\mathrm{PP}) / \mathrm{PA}] \times 100$. Mean startle reactivity across all PA trials was also assessed. Habituation was assessed by comparing mean PA magnitude in the first five trials to mean PA magnitude in the last five trials. A consolidated PPI measure was calculated by averaging percent PPI for each individual subject across all three PP intensities: $\left[\% \mathrm{PPI}_{74 \mathrm{~dB}}+\% \mathrm{PPI}_{78 \mathrm{~dB}}+\% \mathrm{PPI}_{86 \mathrm{~dB}}\right] / 3$. This consolidated measure was used to minimize within-subject variance for correlational analyses. Percent habituation was calculated as follows: [(mean startle amplitude pretestmean startle amplitude posttest)/mean startle amplitude pretest $] \times 100$.

\section{Statistical Analysis}

Groups were compared on demographics and clinical characteristics using independent $t$-tests for continuous variables and Fisher's exact test for categorical variables. PPI group differences were assessed with two-way repeated measures ANOVA, which included between-subjects factors of group (control $v s$ OCD) and gender; and within-subjects repeated measures on percent PPI at each individual PP intensity. Smoking status was not used as an independent 
factor, as OCD subjects and controls were matched for this variable, and the sample contained only two smokers (one OCD subject, one control). Post-hoc independent sample $t$-tests without corrections for multiple comparisons were performed. Group differences in habituation were assessed with an independent $t$-test.

Exploratory analyses examined the association between PPI findings (using the consolidated PPI measure) and clinical features of the OCD subjects. Within the OCD group, Spearman correlations were computed between the consolidated PPI measure and continuous clinical variables (OCD severity as measured by YBOCS; age of onset at which OCD subjects met criteria for diagnosis; current symptom dimensions). Mann-Whitney $U$ was used to compare PPI in OCD patients with and without history of tics.

\section{RESULTS}

\section{Demographics}

Demographics of the 22 OCD and 22 healthy control subjects are shown in Table 1. Because of the a priori matching of the two groups, OCD subjects and healthy controls were not significantly different in gender, ethnicity, smoking status (all $p$ 's $>0.999)$, or age $(p>0.563)$. There also were no group differences in years of education $(p>0.278)$ or verbal IQ (estimated using the NART) $(p>0.993)$.

\section{Clinical Characteristics}

Clinical characteristics of the 22 OCD and 22 healthy control subjects are shown in Table 2 . OCD subjects had clinically significant OCD symptoms, with mean YBOCS of 27 and range from 16 (ie, moderate OCD) to 37 (ie, extreme OCD). All five OCD symptom dimensions were represented (ie, contamination and cleaning, taboo thoughts, doubt and checking, symmetry and ordering, and hoarding), and most patients had symptoms in more than one domain. Only four OCD subjects had symptoms restricted primarily to one domain (contamination and cleaning symptoms, $n=1$;

Table I Demographics of OCD Subjects and Healthy Controls

\begin{tabular}{lcc}
\hline Demographic variables & Healthy controls & OCD subjects \\
\hline $\mathrm{N}$ & 22 & 22 \\
Age (SD) & $31(8.9)$ & $31(9.1)$ \\
Cigarette smokers & 1 & 1 \\
Sex & & \\
Male & 12 & 12 \\
Female & 10 & 10 \\
Ethnicity & & \\
Asian & & 3 \\
Hispanic & 3 & 3 \\
Caucasian & 3 & 16 \\
\hline
\end{tabular}

In all, 22 OCD subjects were individually matched with healthy controls on variables of age, sex, ethnicity, and smoking status. taboo thoughts, $n=2$; symmetry and ordering, $n=1$ ). None of the OCD subjects had pathological grooming behaviors (eg, trichotillomania, skin-picking). Healthy controls had no evidence of OCD symptoms or depression on Y-BOCS or HAM-D, respectively. Three OCD patients had a history of tics: one had a history of transient tic disorder in adolescence; one currently met criteria for chronic tic disorder; and one met criteria for tic disorder NOS (ie, infrequent bouts of eye blinking).

\section{Treatment History}

No patients were taking psychotropic medications at the time of PPI testing. All patients were free of psychotropic medication for at least 4 months. Many patients (14/22; $64 \%$ ) had never taken any psychotropic medications; their mean age at study initiation was $30( \pm 10.2 \mathrm{SD})$. Two patients had only taken benzodiazepines or atypical antipsychotics as needed for acute anxiety. Thus, most OCD patients $(16 / 22 ; 73 \%)$ had never been treated with serotonin reuptake inhibitors. Only one OCD patient had ever received CBT consisting of exposure and response prevention.

\section{PPI of the Acoustic Startle Response}

There were no significant differences in mean startle reactivity between OCD subjects ( $337.7 \pm 57.2$ SEM) and controls $(348.9 \pm 57.2$ SEM) $(p>0.89)$ in PA trials in non-habituation blocks.

Percent PPI was calculated across all PP intensities. In the initial two-way repeated measures ANOVA, there was a significant main effect for group (OCD $v s$ control: $\mathrm{F}$ $(1,40)=6.395 ; p<0.016)$, and a significant main effect for $\mathrm{PP}$ intensity $(\mathrm{F}(2,80)=70.532 ; p<0.0001)$. However, there

Table 2 Clinical Characteristics of OCD Subjects and Healthy Controls

\begin{tabular}{|c|c|c|}
\hline Clinical characteristics & $\begin{array}{l}\text { Healthy controls, } \\
\qquad n=22\end{array}$ & $\begin{array}{c}\text { OCD subjects, } \\
n=22\end{array}$ \\
\hline Y-BOCS & 0 & $27(5.0)$ \\
\hline HAM-D & $0(0.7)$ & $5(4.2)$ \\
\hline Lifetime history of tics & 0 & 3 \\
\hline Age of initial $O C D$ diagnosis & $N / A$ & |6.| (6.9) \\
\hline \multicolumn{3}{|l|}{ DSM-IV Axis I psychiatric comorbidity } \\
\hline No-current or past comorbidity & 22 & 16 \\
\hline Current/past MDD & $0 / 0$ & $0 / 5$ \\
\hline Current social phobia & 0 & । \\
\hline Current panic disorder & 0 & । \\
\hline Current tic disorder & 0 & । \\
\hline Family history of OCD & 0 & 6 \\
\hline \multicolumn{3}{|c|}{$\begin{array}{l}\text { Abbreviations: ASI, anxiety sensitivity index; HAM-D, Hamilton Depression Rating } \\
\text { Scale; OCI-R, obsessive compulsive inventory; OCPD, obsessive compulsive } \\
\text { personality disorder; Y-BOCS, Yale-Brown Obsessive Compulsive Score. } \\
\text { Clinical measures were performed in both OCD subjects and healthy controls. } \\
\text { No OCD symptoms were noted in healthy controls as measured by the } \\
\text { Y-BOCS. SDs are indicated in parentheses. }\end{array}$} \\
\hline
\end{tabular}


was no main effect for gender, no group by gender interaction, and no group by PP intensity interaction (all $p$-values NS). Consistent with previous studies, when gender and the interactions were removed, the final model showed a significant main effect for PP intensity (ie, PPI increased as PP intensity increased) $(\mathrm{F}(2,84)=67.61 ; p<0.0001))$, as has been shown in prior studies (Braff et al, 2001). There was also a main effect for group, with OCD patients having lower PPI than controls $(\mathrm{F}(1,42)=6.48 ; p<0.015)$ (Figure 1). Previous studies reported PPI reductions in OCD patients only at certain PP intensities. To compare the present data with these previous findings, we performed post-hoc comparisons at each PP intensity. Post-hoc tests revealed that OCD subjects had significantly lower percent PPI than healthy controls at all PP intensities: $74 \mathrm{~dB}$ $(\mathrm{t}(42)=2.43 ; \quad p<0.019) ; \quad 78 \mathrm{~dB} \quad(\mathrm{t}(42)=2.15 ; \quad p<0.038)$; $86 \mathrm{~dB}(\mathrm{t}(42)=2.17 ; p<0.036))$. The consolidated PPI measure also demonstrated that OCD patients had significantly lower PPI than healthy controls (mean \pm SEM: healthy controls $=45.4 \pm 4.2$; OCD $=30.1 \pm 4.3 ; t(42)=6.48 ; p<0.015)$.

Percent PPI values for each individual subject across all PP intensities are presented in Figure 2, illustrating the inter-subject variability. Although on average, OCD subjects have decreased percent PPI compared with healthy controls at all PP intensities, there is overlap between the two groups.

\section{Habituation}

Five PA trials were presented at the beginning and end of the PPI session to measure habituation to the startling stimulus. Repeated measures ANOVA of the acoustic startle response demonstrated no significant interaction between group and block $(\mathrm{F}(1,42)<1$; NS). Though there was a significant main effect for block (beginning $v s$ end of session: $\mathrm{F}(1,42)=84.86 ; p<0.0001$, indicating significant habituation over the course of the testing period in both groups), there was no significant main effect for group

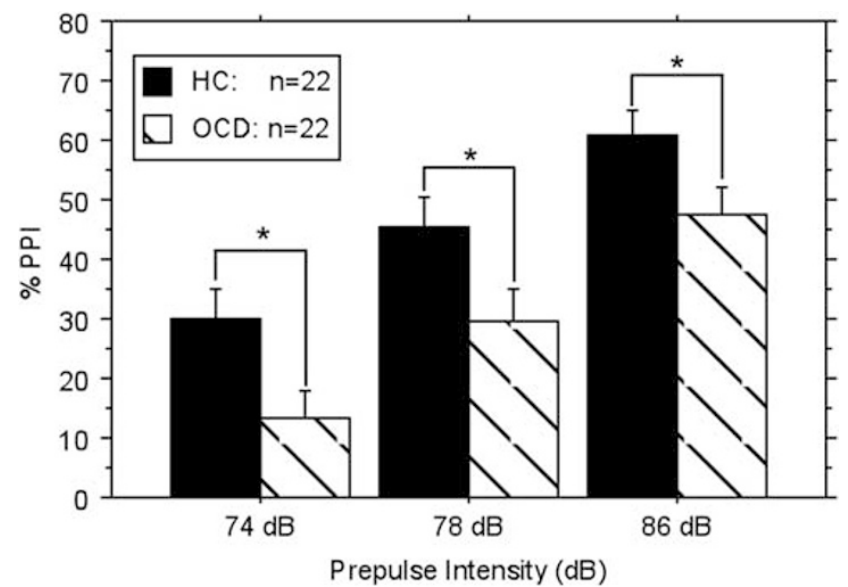

Figure I Percent PPI is significantly lower in OCD subjects. Bar graph demonstrates percent PPI (mean \pm SEM) at 74, 78, and $86 \mathrm{~dB}$ PP intensities. Mean \pm SEM of the mean are as follows: HC: $74 \mathrm{~dB}=29.9$ (5.I); $78 \mathrm{~dB}=45.4 \quad(5.0) ; \quad 86 \mathrm{~dB}=60.9 \quad(4.0) . \quad O C D: \quad 74 \mathrm{~dB}=13.4 \quad$ (4.5); $78 \mathrm{~dB}=29.5(5.5) ; 86 \mathrm{~dB}=47.5$ (4.8). HC: healthy control subjects; OCD: OCD subjects. $*$ Indicates significance at $p<0.02(74 \mathrm{~dB}), p<0.04$ $(78 \mathrm{~dB})$, and $p<0.04(86 \mathrm{~dB})$.
(OCD $v s$ control: $\mathrm{F}(1,42)<1$; NS). When percent habituation was examined (ie, the percentage decrease in the startle response from the beginning to the end of the session), there was no significant difference between OCD subjects and healthy controls $(t(42)=7.8 \mathrm{E}-6$; NS).

\section{Correlations Between PPI Levels and Clinical Ratings}

Exploratory analyses examined the association between PPI findings and clinical features of the 22 OCD patients. Using Spearman rank correlation, there was no significant association (all $p$-values $>0.19$ ) between the consolidated PPI measure and any of the following: OCD severity, age of OCD onset, or any of the five symptom dimensions (ie, hoarding, contamination and cleaning, taboo thoughts, doubt and checking, or symmetry and ordering). However, OCD patients with any history of tics $(n=3)$ had a trend towards lower percent PPI than OCD patients without a history of tics (Mann-Whitney $U ; p<0.077$ ).

\section{DISCUSSION}

Our data demonstrate that unmedicated OCD patients have PPI deficits across three PP intensities compared with matched healthy controls. No differences were seen in either habituation or baseline startle response between the two groups. Exploratory analyses of clinical correlations within the OCD group suggested that OCD subjects with a history of tics were those most likely to have PPI deficits.

Our results confirm the findings of Swerdlow et al (1993) and Hoenig et al (2005), and extend their work in several ways. First, we demonstrate that unmedicated OCD patients have PPI deficits. This result is consistent with Hoenig et al (2005), who found in a post-hoc analysis that those OCD patients who were free of medication $(n=4)$ had lower PPI (ie, larger PPI deficits) than the group mean $(n=30)$; the rest of the group was taking medications $(n=26: 12$ SSRIs; 4 TCAs; 2 TCA + SSRI; 8 antipsychotics + SSRI). Prior clinical and preclinical studies have suggested that psychotropic medications used to treat OCD have effects on PPI,

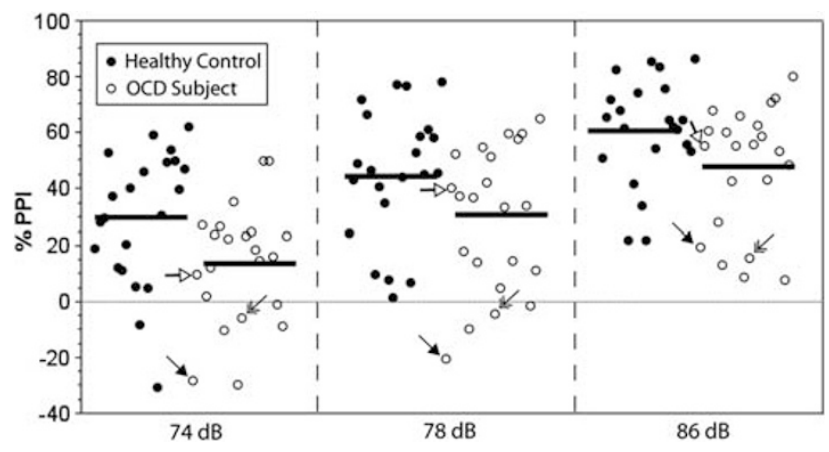

Figure 2 Percent PPI for each individual subject demonstrates intersubject variation. Scatter-plot indicates percent PPI for each individual subject at PP intensities of 74, 78, and $86 \mathrm{~dB}$. Closed circles indicate healthy controls $(n=22)$ and open circles indicate OCD subjects $(n=22)$. Black bars indicate the mean percent PPI at each PP intensity. The three individuals with tics are highlighted with arrows; each individual subject is assigned a different arrowhead (filled, open, or double) that can be tracked across all three PP intensities. 
including both SSRIs (Quednow et al, 2004; Shanahan et al, 2009) and antipsychotics (Swerdlow et al, 1994). Our study eliminated this confound. Second, we observed PPI deficits across all PP intensities $(74,78$, and $86 \mathrm{~dB})$, whereas deficits were observed previously only at $74 \mathrm{~dB}$ (Swerdlow et al, 1993) or at $86 \mathrm{~dB}$ (Hoenig et al, 2005). It is possible that eliminating the confound of medication may have helped to reveal group differences at all PP intensities. Third, we also reduced the potential confound of hormonal status in females by only measuring PPI in early follicular phase of the menstrual cycle, and excluding subjects who used hormonal methods of birth control. This approach was used because prior studies have shown that PPI levels fluctuate across the menstrual cycle (Swerdlow et al, 1997; Jovanovic et al, 2004). Finally, in our study, OCD subjects were individually paired with healthy controls, who were matched on age, ethnicity, and gender; and only one subject in each group smoked cigarettes. This careful matching may have allowed us to minimize variability in our sample, thereby increasing our power to detect group differences.

de Leeuw et al (2010) also examined PPI in unmedicated OCD subjects and did not find PPI deficits at any PP intensity. Methodological differences between our study and theirs might explain this difference in findings. First, our subjects had their eyes open during PPI measurement, whereas subjects in de Leeuw et al (2010) had their eyes closed. In our experience, measuring PPI in subjects with closed eyes can lead to significant variability in startle measurements because of subjects falling asleep. Second, we had a 4-month medication-free period for OCD patients, whereas de Leeuw et al (2010) had a 4-week medication-free period for fluoxetine, and 2 weeks for all other medications. Moreover, we confirmed that OCD and healthy control subjects all had negative urine toxicology screens on the day of testing. This was important as we found that self-report of illicit substance use was unreliable in those we screened; several potential healthy control subjects were excluded because of positive tests for benzodiazepines, amphetamine, and marijuana, all of which can impact PPI (Geyer et al, 2001). Finally, other clinical characteristics of the samples may have differed. Our healthy controls had never taken any psychiatric medications and had no family history of OCD (of importance because PPI deficits could constitute an endophenotype); only 6 of our 22 OCD patients had a history of any Axis I psychiatric illness other than OCD. These clinical characteristics were not described in de Leeuw et al (2010).

PPI deficits are thought to reflect abnormal processing and integration of sensory and motor information (Butler et al, 1990; Geyer et al, 2001). Animal studies indicate that the brain circuit modulating PPI involves the corticostriatal-pallido-pontine (CSPP) loop (Swerdlow et al, 1999, 2001; Geyer et al, 2001; Baldan Ramsey et al, 2011). Our findings thus support the idea that OCD patients with PPI deficits may have difficulty gating sensory information or inhibiting subsequent responses, which may manifest as a deficiency in 'filtering out' obsessive thoughts or compulsive urges. The presence of PPI deficits also suggests that OCD patients have CSPP circuit abnormalities, which is further supported by imaging studies (Maia et al, 2008).

Although we observe a significant group difference across PP intensities, individual data demonstrate substantial overlap in individual PPI values, illustrating that not all OCD patients had PPI deficits. PPI deficits are therefore not a characteristic that unifies all OCD subjects. Many neurophysiological measures associated with neuropsychiatric disease also show substantial overlap between individual patients and controls despite mean group differences (Kanai and Rees, 2011). Through exploratory analyses, we thus attempted to identify clinical factors shared by the patients with lower percent PPI. The only clinical factor that demonstrated an association with PPI deficits was the presence of a history of tics. If replicated, this finding would be consistent with the fact that PPI deficits have been demonstrated in Tourette's syndrome (Castellanos et al, 1996; Swerdlow et al, 2001). In addition, some have advocated for a tic-related subtype of OCD categorized by presence of tics either in the OCD proband or in a first-degree relative (Leckman et al, 2010). This tic-related subtype has been found to have increased familial transmission, a different clinical course, and potentially different underlying genetic factors (Leckman et al, 2010). In addition, there is evidence that pediatric OCD patients with a history of tics have higher baseline prolactin levels than those without tics (Hanna et al, 1991). We speculate that PPI deficits might characterize tic-related OCD. If so, further investigation of the brain mechanisms underlying PPI deficits in OCD may shed light on the neurobiology underlying this subtype.

Our findings should be considered in light of two limitations. First, we did not directly measure hormonal status in female subjects, and thus relied on self-report. Second, our sample size provided limited power to detect small correlations between PPI deficits and clinical measures. Thus, the finding of lower PPI in OCD patients with a history of tics should be considered preliminary.

In summary, our study demonstrated significant PPI deficits in unmedicated OCD patients. This result suggests that PPI deficits arise from circuit abnormalities inherent to OCD pathology, and not from medication effects. At the same time, PPI deficits are a general biomarker of corticostriatal-pallidal circuit pathology. This likely explains the presence of PPI deficits in disorders that are often comorbid with OCD and also have cortico-striatal-pallidal circuit pathology (eg, schizophrenia, autism, and Tourette's syndrome). In combination with translatable tasks that probe other relevant circuitry (eg, fear circuitry), PPI can therefore now be used to validate animal models of OCD. In addition, our results provide the foundation for examining the effects of OCD treatment (medications or therapy) on PPI. Such studies would allow a determination of whether PPI is (a) an endophenotype or (b) a state-dependent measure that can be used to track changes in neural circuitry related to treatment response. Finally, our findings suggest that OCD patients with a history of tics may have greater impairment in sensorimotor gating than the general OCD population. Future studies thus need to determine whether PPI deficits are a reliable feature of tic-related OCD.

\section{ACKNOWLEDGEMENTS}

We would like to thank Dr James Bender, Rena Staub, and Liane Hunter for performing patient recruitment and 
screening; Page van Meter and Andrew Schmidt for database management; Kimberly Glazier for assistance with data processing; Dr Donna Vermes, Dr Rafael Campeas, Dr Anthony Pinto, and Dr Carolyn Rodriguez for assistance with clinical ratings; and Dr Tobias Teichert for statistical consultation. This work was supported by an NIH T32 research fellowship, NIH K08 MH087718-01A1, and a Louis V Gerstner, Jr Scholars Grant to S.E.A.; by NIH R24 MH080022 and NIH K24 MH091555 to H.B.S.; and by the New York State Office of Mental Health.

\section{DISCLOSURE}

In the last 3 years, Dr Simpson has received medication at no-cost from Janssen Pharmaceuticals for an NIMH-funded study, consulting fees from Pfizer for advice regarding the medication Lyrica, and research funds from Neuropharm and from Transcept Pharmaceuticals to conduct clinical trials of novel medications for OCD. Dr Geyer has received consulting compensation from Acadia, Addex, Cerca, Medivation, Merck, Omeros, Takeda, and Teva, and holds an equity interest in San Diego Instruments. Dr Risbrough has received consulting fees from Arena, Cenomed, and Ferring Pharmaceuticals. Dr Geyer has research grant support from Intracellular Therapeutics, Johnson \& Johnson, NIDA, NIMH, and the US Veteran's Administration VISN 22 Mental Illness Research, Education, and Clinical Center. The remaining authors declared no conflict of interest.

\section{REFERENCES}

Baldan Ramsey LC, Xu M, Wood N, Pittenger C (2011). Lesions of the dorsomedial striatum disrupt prepulse inhibition. Neuroscience 180: 222-228.

Braff DL, Geyer MA, Swerdlow NR (2001). Human studies of prepulse inhibition of startle: normal subjects, patient groups, and pharmacological studies. Psychopharmacology (Berl) 156: 234-258.

Butler RW, Braff DL, Rausch JL, Jenkins MA, Sprock J, Geyer MA (1990). Physiological evidence of exaggerated startle response in a subgroup of Vietnam veterans with combat-related PTSD. Am J Psychiatry 147: 1308-1312.

Castellanos FX, Fine EJ, Kaysen D, Marsh WL, Rapoport JL, Hallett M (1996). Sensorimotor gating in boys with Tourette's syndrome and ADHD: preliminary results. Biol Psychiatry 39: 33-41.

de Leeuw AS, Oranje B, van Megen HJ, Kemner C, Westenberg HG. (2010). Sensory gating and sensorimotor gating in medicationfree obsessive-compulsive disorder patients. Int Clin Psychopharmacol 25: 232-240.

Dickel DE, Veenstra-VanderWeele J, Cox NJ, Wu X, Fischer DJ, Van Etten-Lee $M$ et al (2006). Association testing of the positional and functional candidate gene SLC1A1/EAAC1 in early-onset obsessive-compulsive disorder. Arch Gen Psychiatry 63: 778-785.

Ekselius L, Lindström E, von Knorring L, Bodlund O, Kullgren G (1994). SCID II interviews and the SCID Screen questionnaire as diagnostic tools for personality disorders in DSM-III-R. Acta Psychiatr Scand 90: 120-123.

Fendt M, Li L, Yeomans JS. (2001). Brain stem circuits mediating prepulse inhibition of the startle reflex. Psychopharmacology (Berl) 156: 216-224.
First MB, Spitzer RL (1996). Structured Clinical Interview for DSM-IV Axis I Disorders-Patient Edition. Biometrics Research Department, New York State Psychiatric Institute: New York, NY.

Geyer MA, Dulawa SC (2003). Assessment of murine startle reactivity, prepulse inhibition, and habituation. Curr Protoc Neurosci. Chapter 8: Unit 817.

Geyer MA, Krebs-Thomson K, Braff DL, Swerdlow NR (2001). Pharmacological studies of prepulse inhibition models of sensorimotor gating deficits in schizophrenia: a decade in review. Psychopharmacology (Berl) 156: 117-154.

Goodman WK, Price LH, Rasmussen SA, Mazure C, Delgado P, Heninger GR et al (1989a). The Yale-Brown Obsessive Compulsive Scale. II validity. Arch Gen Psychiatry 46: 1012-1016.

Goodman WK, Price LH, Rasmussen SA, Mazure C, Fleischmann RL, Hill CL et al (1989b). The Yale-Brown Obsessive Compulsive Scale. I development, use, and reliability. Arch Gen Psychiatry 46: 1006-1011.

Graham FK (1975). Presidential address, 1974. The more or less startling effects of weak prestimulation. Psychophysiology 12: 238-248.

Hamilton M (1960). A rating scale for depression. J Neurol Neurosurg Psychiatry 23: 56-62.

Hammer A, Kordon A, Heldmann M, Zurowski B, Münte TF (2009). Brain potentials of conflict and error-likelihood following errorful and errorless learning in obsessive-compulsive disorder. PLoS One 4: e6553.

Hanna GL, McCracken JT, Cantwell DP (1991). Prolactin in childhood obsessive-compulsive disorder: clinical correlates and response to clomipramine. J Am Acad Child Adolesc Psychiatry 30: $173-178$.

Hoenig K, Hochrein A, Quednow BB, Maier W, Wagner M (2005). Impaired prepulse inhibition of acoustic startle in obsessivecompulsive disorder. Biol Psychiatry 57: 1153-1158.

Huyser C, Veltman DJ, de Haan E, Boer F (2009). Paediatric obsessive-compulsive disorder, a neurodevelopmental disorder? Evidence from neuroimaging. Neurosci Biobehav Rev 33: 818-830.

Jovanovic T, Szilagyi S, Chakravorty S, Fiallos AM, Lewison BJ, Parwani A et al (2004). Menstrual cycle phase effects on prepulse inhibition of acoustic startle. Psychophysiology 41: 401-406.

Kanai R, Rees G (2011). The structural basis of inter-individual differences in human behaviour and cognition. Nat Rev Neurosci 12: 231-242.

Kessler RC, Berglund P, Demler O, Jin R, Merikangas KR, Walters EE (2005). Lifetime prevalence and age-of-onset distributions of DSM-IV disorders in the National Comorbidity Survey Replication. Arch Gen Psychiatry 62: 593-602.

Koch M, Schnitzler HU (1997). The acoustic startle response in rats-circuits mediating evocation, inhibition and potentiation. Behav Brain Res 89: 35-49.

Leckman JF, Denys D, Simpson HB, Mataix-Cols D, Hollander E, Saxena $S$ et al (2010). Obsessive-compulsive disorder: a review of the diagnostic criteria and possible subtypes and dimensional specifiers for DSM-V. Depress Anxiety 27: 507-527.

Leckman JF, Riddle MA, Hardin MT, Ort SI, Swartz KL, Stevenson J et al (1989). The Yale Global Tic Severity Scale: initial testing of a clinician-rated scale of tic severity. J Am Acad Child Adolesc Psychiatry 28: 566-573.

Maia TV, Cooney RE, Peterson BS (2008). The neural bases of obsessive-compulsive disorder in children and adults. Dev Psychopathol 20: 1251-1283.

Murray CJ, Lopez AD (1996). Evidence-based health policylessons from the Global Burden of Disease Study. Science 274: 740-743.

Nelson HE, O'Connell A (1978). Dementia: the estimation of premorbid intelligence levels using the New Adult Reading Test. Cortex 14: 234-244. 
Pinto A, Eisen JL, Mancebo MC, Greenberg BD, Stout RL, Rasmussen SA (2007). Taboo thoughts and doubt/checking: a refinement of the factor structure for obsessive-compulsive disorder symptoms. Psychiatry Res 151: 255-258.

Pinto A, Greenberg BD, Grados MA, Bienvenu III OJ, Samuels JF, Murphy DL et al (2008). Further development of YBOCS dimensions in the OCD Collaborative Genetics study: symptoms vs. categories. Psychiatry Res 160: 83-93.

Pinto A, Greenberg BD, Murphy DL, Nestadt G, Rasmussen SA (2009). Using individual items to clarify OCD symptom structure: the case for five factors. Am J Psychiatry 166: 728-729; author reply 729-731.

Quednow BB, Kühn KU, Stelzenmuelle R, Hoenig K, Maier W, Wagner M (2004). Effects of serotonergic and noradrenergic antidepressants on auditory startle response in patients with major depression. Psychopharmacology (Berl) 175: 399-406.

Robins LN, Helzer JE, Weissman MM, Orvaschel H, Gruenberg E, Burke Jr JD et al (1984). Lifetime prevalence of specific psychiatric disorders in three sites. Arch Gen Psychiatry 41: 949-958.

Shanahan NA, Holick Pierz KA, Masten VL, Waeber C, Ansorge M, Gingrich JA et al (2009). Chronic reductions in serotonin transporter function prevent 5-HT1B-induced behavioral effects in mice. Biol Psychiatry 65: 401-408.

Simpson HB, Slifstein M, Bender Jr J, Xu X, Hackett E, Maher MJ et al (2011). Serotonin 2A Receptors in Obsessive-Compulsive Disorder: A Positron Emission Tomography Study with [(11)C]MDL 100907. Biol Psychiatry 70: 897-904.
Swerdlow NR, Benbow CH, Zisook S, Geyer MA, Braff DL (1993). A preliminary assessment of sensorimotor gating in patients with obsessive compulsive disorder. Biol Psychiatry 33: 298-301.

Swerdlow NR, Braff DL, Geyer MA (1999). Cross-species studies of sensorimotor gating of the startle reflex. Ann N Y Acad Sci 877: 202-216.

Swerdlow NR, Braff DL, Taaid N, Geyer MA (1994). Assessing the validity of an animal model of deficient sensorimotor gating in schizophrenic patients. Arch Gen Psychiatry 51: 139-154.

Swerdlow NR, Geyer MA (1998). Using an animal model of deficient sensorimotor gating to study the pathophysiology and new treatments of schizophrenia. Schizophr Bull 24: 285-301.

Swerdlow NR, Geyer MA, Braff DL (2001). Neural circuit regulation of prepulse inhibition of startle in the rat: current knowledge and future challenges. Psychopharmacology (Berl) 156: 194-215.

Swerdlow NR, Hartman PL, Auerbach PP (1997). Changes in sensorimotor inhibition across the menstrual cycle: implications for neuropsychiatric disorders. Biol Psychiatry 41: 452-460.

Swerdlow NR, Karban B, Ploum Y, Sharp R, Geyer MA, Eastvold A (2001). Tactile prepuff inhibition of startle in children with Tourette's syndrome: in search of an 'fMRI-friendly' startle paradigm. Biol Psychiatry 50: 578-585.

Weissman MM, Wickramaratne P, Adams P, Wolk S, Verdeli H, Olfson M (2000). Brief screening for family psychiatric history: the family history screen. Arch Gen Psychiatry 57: $675-682$. 